\title{
Traveling Wavefronts on Reaction Diffusion Systems with Spatio-Temporal Delays
}

\author{
Xinli Han ${ }^{1}$, Lijun Pan ${ }^{2}$ \\ ${ }^{1}$ College of Science, Nanjing University of Posts and Telecommunications, Nanjing, China \\ ${ }^{2}$ Department of Mathematics, Nanjing University of Aeronautics and Astronautics, Nanjing, China \\ Email: xinlihan@126.com,98010149@163.com
}

Received November 1, 2012; revised January 1, 2013; accepted January 9, 2013

Copyright (C) 2013 Xinli Han, Lijun Pan. This is an open access article distributed under the Creative Commons Attribution License, which permits unrestricted use, distribution, and reproduction in any medium, provided the original work is properly cited.

\begin{abstract}
By using Schauder's Fixed Point Theorem, we study the existence of traveling wave fronts for reaction-diffusion systems with spatio-temporal delays. In our results, we reduce the existence of traveling wave fronts to the existence of an admissible pair of upper solution and lower solution which are much easier to construct in practice.
\end{abstract}

Keywords: Schauder's Fixed Point Theorem; Traveling Wave Fronts; Reaction-Diffusion; Spatio-Temporal Delays

\section{Introduction}

Traveling wave solutions, usually characterized as solutions invariant with respect to translation in space, have attracted much attention due to their significant nature in science and engineering [1-18]. In which, the theory of wave fronts of reaction diffusion systems is an important part, and its history traces back to the so-called FisherKPP equation, the celebrated mathematical works by P. A. Fisher and by Kolmogorov, Petrovskii and Piscunov. Since then, lots of papers are devoted to the study of traveling wave solutions of reaction diffusion systems, and various research methods come forth.

The present paper is mainly devoted to tackle the existence of traveling wave front solutions of the following reaction diffusion system with spatial-temporal delays and with some zero-diffusive coefficients,

$$
\begin{aligned}
\frac{\partial U(t, x)}{\partial t}= & D \frac{\partial^{2} U(t, x)}{\partial x^{2}} \\
& +F\left(\left(g_{1} * U\right)(t, x), \cdots,\left(g_{m} * U\right)(t, x)\right)
\end{aligned}
$$

where $t \geq 0, x \in \mathbb{R} ; \quad D=\operatorname{diag}\left(d_{1}, \cdots, d_{n}\right), \sum_{i=1}^{n} d_{i}^{2} \neq 0$, $d_{i} \geq 0, U(t, x)=\left(u_{1}(t, x), \cdots, u_{n}(t, x)\right)^{\mathrm{T}}$, $F=\left(f_{1}, \cdots, f_{n}\right)^{\mathrm{T}} \in\left(\mathbb{R}^{2}, \mathbb{R}^{n}\right)$, $\left(g_{j} * U\right)(t, x)=\int_{-\infty}^{t} \int_{-\infty}^{+\infty} g_{j}(t-s, x-y) U(s, y) \mathrm{d} y \mathrm{~d} s$, $j=1, \cdots, m$.

Here the kernels of convolutions $g_{j} * U(j=1, \cdots, m)$, satisfy

$$
\begin{aligned}
& \int_{0}^{+\infty} \int_{-\infty}^{+\infty} g_{j}(s, y) \mathrm{d} y \mathrm{~d} s=1, \\
& g_{j}(t, x) \geq 0,(t, x) \in \mathbb{R}_{+0} \times \mathbb{R} .
\end{aligned}
$$

And the kernels used frequently in the reference are as follows

1) $g_{j}(t, x)=\delta(t) \delta(x)$;

2) $g_{j}(t, x)=\delta(t) p_{j}(x)$;

3) $g_{j}(t, x)=\delta\left(t-\tau_{j}\right) \delta(x)$;

4) $g_{j}(t, x)=q_{j}(t) \delta(x)$;

5) $g_{j}(t, x)=\delta\left(t-\tau_{j}\right) p_{j}(x)$.

The remaining part of this paper is organized as follows. In the next section, some preliminaries are given. In Section 3, we state and prove the main result of this paper.

\section{Preliminaries}

A traveling wave solution of (1.1) is a special translation invariant solution of the form $U(t, x)=\Phi(x+c t)$, where $\Phi \in C^{2}\left(R, R^{n}\right)$ is the profile of the wave that propagates through the one-dimensional spatial domain at a constant velocity $c>0$. If $\Phi$ is monotone and satisfies the asymptotic boundary conditions $\lim _{s \rightarrow-\infty} \Phi(s)=U^{-}$ and $\lim _{s \rightarrow+\infty} \Phi(s)=U^{+}, \quad U(t, x)=\Phi(x+c t)$ is called a 
wave front of (1.1), where $U^{-}=\left(u_{1}^{-}, \cdots, u_{n}^{-}\right)^{\mathrm{T}}$, $U^{+}=\left(u_{1}^{+}, \cdots, u_{n}^{+}\right)^{\mathrm{T}} \in \mathbb{R}^{n}, U^{-}<U^{+}$, and $U, U^{+}$are equilibria of system (1.1). If $Y<Z$, we also denote

$$
B C[Y, Z]:=\left\{\Phi \in C\left(\mathbb{R}, \mathbb{R}^{n}\right): Y \leq \Phi(t) \leq Z, t \in \mathbb{R}\right\}
$$

Let $\|\cdot\|$ be the supremum norm in $\mathbb{R}^{n}$ and $C\left([a, b] ; \mathbb{R}^{n}\right)$, and

$$
\begin{aligned}
& B C\left(\mathbb{R} ; \mathbb{R}^{n}\right):=\left\{\Phi \in C\left(\mathbb{R} ; \mathbb{R}^{n}\right): \sup _{t \in \mathbb{R}}\|\Phi(t)\|<\infty\right\} \\
& B C_{\mu}\left(\mathbb{R} ; \mathbb{R}^{n}\right):=\left\{\Phi \in C\left(\mathbb{R} ; \mathbb{R}^{n}\right): \sup _{t \in \mathbb{R}}\|\Phi(t)\| e^{-\mu|t|}<\infty\right\} \\
& B C^{2}\left(\mathbb{R} ; \mathbb{R}^{n}\right):=\left\{\Phi: \max _{d_{i}>0} \sup _{t \in \mathbb{R}}\left\{\left|\phi_{i}^{\prime \prime}(t)\right|\right\}<\infty, \Phi, \Phi^{\prime} \in B C\left(\mathbb{R} ; \mathbb{R}^{n}\right) \sup _{t \in \mathbb{R}} \text { and } \phi^{\prime \prime} \in C(\mathbb{R} ; \mathbb{R}), \text { here, } d_{i}>0\right\}
\end{aligned}
$$

where $\mu$ will be given in the next section. Obviously, $B C\left(\mathbb{R} ; \mathbb{R}^{n}\right), \quad B C_{\mu}\left(\mathbb{R} ; \mathbb{R}^{n}\right)$ and $B C^{2}\left(\mathbb{R} ; \mathbb{R}^{n}\right)$ are Banach spaces respectively with the norms

$$
\begin{aligned}
& \|\Phi\|_{0}:=\sup _{t \in \mathbb{R}}\|\Phi(t)\|, \Phi \in B C\left(\mathbb{R} ; \mathbb{R}^{n}\right), \\
& \|\Phi\|_{\mu}:=\sup _{t \in \mathbb{R}}\|\Phi(t)\| \mathrm{e}^{-\mu|t|}, \Phi \in B C_{\mu}\left(\mathbb{R} ; \mathbb{R}^{n}\right), \\
& \|\Phi\|_{2}:=\max _{d_{i}>0}\left\{\left\|\Phi_{0}\right\|,\left\|\Phi_{0}^{\prime}\right\| \sup _{t \in \mathbb{R}}\left\{\left|\phi_{i}^{\prime \prime}(t)\right|\right\}\right\}, \Phi \in B C^{2}\left(\mathbb{R} ; \mathbb{R}^{n}\right) .
\end{aligned}
$$

Substituting $U(t, x)=\Phi(x+c t)$ into (1.1) and denoting still by $t$ the traveling coordinate $x+c t$, we obtain the corresponding wave equations

$$
\begin{aligned}
& c \Phi^{\prime}(t)=D \Phi^{\prime \prime}(t)+F\left(\left(g_{1} * \Phi\right)(t), \cdots,\left(g_{m} * \Phi\right)(t)\right), \\
& t \in \mathbb{R},
\end{aligned}
$$

where $c>0$ is velocity, $\sum_{i=1}^{n} d_{i}^{2} \neq 0, d_{i} \geq 0, \quad i=1, \cdots, n$; and

$$
\left(g_{j} * \Phi\right)(t)=\int_{0}^{+\infty} \int_{-\infty}^{+\infty} g_{j}(s, y) \Phi(t-y-c s) \mathrm{d} y \mathrm{~d} s,
$$

Without loss of generality, we assume $U^{-}=\mathbf{0}=(0, \cdots, 0)^{\mathrm{T}}, \quad U^{+}=\boldsymbol{K}=\left(K_{1}, \cdots, K_{n}\right)^{\mathrm{T}}$, the asymptotic boundary conditions are replaced by

$$
\lim _{t \rightarrow-\infty} \Phi(t)=0, \lim _{t \rightarrow+\infty} \Phi(t)=\boldsymbol{K}
$$

In the following, we list the basic assumptions of this paper:

$\left(\mathrm{A}_{1}\right) \quad F(\mathbf{0}, \cdots, \mathbf{0})=F(\boldsymbol{K}, \cdots, \boldsymbol{K})=0$.

$\left(\mathrm{A}_{2}\right)$ There exist positive constants $\sigma_{j} \leq 1$ and $L_{j}$, such that for all $Y_{j}, Z_{j} \in[\mathbf{0}, \boldsymbol{K}], j=1, \cdots, m$,

$$
\left\|F\left(Y_{1}, \cdots, Y_{m}\right)-F\left(Z_{1}, \cdots, Z_{m}\right)\right\| \leq \sum_{j=1}^{m} L_{j}\left\|Y_{j}-Z_{j}\right\|^{\sigma_{j}} .
$$

$\left(\mathrm{A}_{3}\right)$ There exists an constant $\beta>0$ such that for $j=1, \cdots, m$, and $\Phi \in B C[-\boldsymbol{K}, \boldsymbol{K}]$,

$$
\int_{0}^{+\infty} \int_{-\infty}^{+\infty} g_{j}(s, y)\|\Phi(t-y-c s)\| \mathrm{d} y \mathrm{~d} s \leq \beta\|\Phi\|_{\mu} \mathrm{e}^{\mu|t|} .
$$

$\left(\mathrm{A}_{4}\right)$ One of the following two cases holds.

$\left(\mathrm{A}_{4}^{1}\right) \quad \int_{0}^{+\infty} g_{j}(t, x) \mathrm{d} t$ is uniformly convergent for $x \in[-a, a]$, where $a>0$, i.e., for given $\varepsilon>0$, there exists $b>0$ s.t. $\int_{b}^{+\infty} g_{j}(t, x) \mathrm{d} t<\varepsilon$ for all $x \in[-a, a]$, $j=1, \cdots, m$.

$\left(\mathrm{A}_{4}^{2}\right) \quad \int_{-\infty}^{+\infty} g_{j}(t, x) \mathrm{d} x$ is uniformly convergent for $t \in[0, b]$, where $b>0$, i.e., for given $\varepsilon>0$, there exists $a>0$, s.t. $\int_{a}^{+\infty} g_{j}(t, x) \mathrm{d} x<\varepsilon$ and $\int_{-\infty}^{a} g_{j}(t, x) \mathrm{d} x<\varepsilon$ for all $t \in[0, b], \quad j=1, \cdots, m$.

$\left(\mathrm{A}_{5}\right)$ There exists a matrix $\gamma=\operatorname{diag}\left(\gamma_{1}, \cdots, \gamma_{n}\right), \gamma_{i} \geq 0$, s.t.

$$
\begin{aligned}
& F\left(\left(g_{1} * \Phi\right)(t), \cdots,\left(g_{m} * \Phi\right)(t)\right)+\gamma \Phi(t) \\
& \geq F\left(\left(g_{1} * \Psi\right)(t), \cdots,\left(g_{m} * \Psi\right)(t)\right)+\gamma \Psi(t)
\end{aligned}
$$

where $t \in \mathbb{R}$ and $\Phi, \Psi \in C\left(\mathbb{R}, \mathbb{R}^{n}\right)$ satisfy $\hat{\mathbf{0}} \leq \Psi \leq \Phi \leq \hat{\boldsymbol{K}}$, here and in the sequel, $\hat{U}$ denote the constant vector function on $\mathbb{R}$, taking the value $U=\left(u_{1}, \cdots, u_{n}\right)^{\mathrm{T}} \in \mathbb{R}^{n}$.

At the end of this section, we give the following two useful lemmas.

Lemma 2.1. [8] Let $x: \mathbb{R}_{+0} \rightarrow \mathbb{R}$ be a differentiable function. If $\liminf _{t \rightarrow \infty} x(t)<\lim _{t \rightarrow \infty} \sup x(t)$, there are sequences $\left\{s_{n}\right\}_{n=1}^{\infty}$ and $\left\{t_{n}\right\}_{n=1}^{\infty}$ with $\lim _{n \rightarrow \infty} s_{n}=\lim _{n \rightarrow \infty} t_{n}=\infty$ s.t.

$$
\left\{\begin{array}{l}
\lim _{n \rightarrow \infty} x\left(s_{n}\right)=\lim _{t \rightarrow \infty} \inf x(t) \text { and } x^{\prime}\left(s_{n}\right)=0, \\
\lim _{n \rightarrow \infty} x\left(t_{n}\right)=\lim _{t \rightarrow \infty} \sup x(t) \text { and } x^{\prime}\left(t_{n}\right)=0 .
\end{array}\right.
$$

Lemma 2.2. $[3,8]$ Let $a \in \mathbb{R}$, and $x:[a,+\infty) \rightarrow \mathbb{R}$ be a differentiable function. If $\lim _{t \rightarrow \infty} x(t)$ exists (finite) and the derivative function $x^{\prime}(t)$ is uniformly continuous on $[a,+\infty)$ then $\lim _{t \rightarrow \infty} x^{\prime}(t)=0$. 


\section{Main Theorem}

First, we introduce the definition an upper-lower solution of wave Equations (2.1)

Definition 3.1. A continuous function $\bar{\rho}=\left(\bar{\rho}_{1}, \cdots, \bar{\rho}_{n}\right)$ $\mathbb{R} \rightarrow \mathbb{R}^{n}$ is called an upper solution of (2.1), if $\bar{\rho}_{i}^{\prime}(t)$ and $\bar{\rho}_{i}^{\prime \prime}(t)$ (if $d_{i}>0$ ) exist almost everywhere and they are essentially bounded, and $\bar{\rho}_{i}$ satisfies almost everywhere on $\mathbb{R}$

$$
c \bar{\rho}_{i}^{\prime}(t) \geq d_{i} \bar{\rho}_{i}^{\prime \prime}(t)+f_{i}\left(\left(g_{1} * \bar{\rho}\right)(t), \cdots,\left(g_{m} * \bar{\rho}\right)(t)\right)
$$

A lower solution $\rho=\left(\underline{\rho}_{1}, \cdots, \rho_{n}\right)$ of (2.1) can be given in a similar way by reversing the inequality in (3.1).

For wave equations (2.1), we have the following results.

Proposition 3.1. Assume $\left(A_{2}\right)$ holds. If wave equations (2.1) have a monotone solution $\Phi \in C\left(\mathbb{R} ; \mathbb{R}^{n}\right)$ satisfying $\lim _{t \rightarrow-\infty} \Phi(t)=V_{-}$and $\lim _{t \rightarrow-\infty} \Phi(t)=V_{+}$, where
$0 \leq V_{-} \leq V_{+} \leq K, \quad V_{-}, V_{+} \in \mathbb{R}^{n}$ then

$$
F\left(\left(g_{1} * \Phi\right)(t), \cdots,\left(g_{m} * \Phi\right)(t)\right)
$$

is uniformly continuous in $\mathbb{R}$.

Proof. It is not difficult to show $\Phi(t)$ is uniformly continuous in $\mathbb{R}$, then we know that $\forall \varepsilon>0$ sufficiently small $\left(\varepsilon<m L, L=\max _{1 \leq j \leq l} L_{j}\right)$, there is a constant $\delta$, s.t. for $t_{1}, t_{2} \in \mathbb{R}$ and $\left|t_{1}-t_{2}\right|<\delta$,

$$
\left\|\Phi\left(t_{1}-y-c s\right)-\Phi\left(t_{2}-y-c s\right)\right\|<(\varepsilon / m L)^{1 / \sigma},
$$

where $s \geq 0, \quad y \in \mathbb{R}, \quad \sigma=\min _{1 \leq j \leq l} \sigma_{j}$, and $\sigma_{j}(j=1, \cdots, m)$ are given in $\left(\mathrm{A}_{2}\right)$. In addition, we can obtain

$$
\begin{aligned}
& \left(g_{i} * \Phi\right)\left(t_{1}\right)-\left(g_{i} * \Phi\right)\left(t_{2}\right) \\
& \left(g_{i} * \Phi\right)\left(t_{1}\right)-\left(g_{i} * \Phi\right)\left(t_{2}\right) \in[0, K], j=1, \cdots, m .
\end{aligned}
$$

Then by $\left(\mathrm{A}_{2}\right)$, we have

$$
\begin{aligned}
& \left\|F\left(\left(g_{1} * \Phi\right)\left(t_{1}\right), \cdots,\left(g_{m} * \Phi\right)\left(t_{1}\right)\right)-F\left(\left(g_{1} * \Phi\right)\left(t_{2}\right), \cdots,\left(g_{m} * \Phi\right)\left(t_{2}\right)\right)\right\| \\
& \leq \sum_{j=1}^{m} L_{j}\left[\int_{0}^{+\infty} \int_{-\infty}^{+\infty} g_{j}(s, y)\left\|\Phi\left(t_{1}-y-c s\right)-\Phi\left(t_{2}-y-c s\right)\right\| \mathrm{d} y \mathrm{~d} s\right]^{\sigma_{j}} \leq \varepsilon
\end{aligned}
$$

Thus, $F\left(\left(g_{1} * \Phi\right)(t), \cdots,\left(g_{m} * \Phi\right)(t)\right)$, is uniformly continuous in $\mathbb{R}$. This completes the proof of the proposition.

Proposition 3.2. Assume $\left(\mathrm{A}_{2}\right)$ and $\left(\mathrm{A}_{4}\right)$ hold. If wave equations (2.1) have a monotone solution $\Phi \in C\left(\mathbb{R} ; \mathbb{R}^{n}\right)$ satisfying $\lim _{t \rightarrow-\infty} \Phi(t)=V_{-}$and $\lim _{t \rightarrow \infty} \Phi(t)=V_{+}$, where $0 \leq V_{-} \leq V_{+} \leq K, V_{-}, V_{+} \in \mathbb{R}^{n}$, then

$$
F\left(V_{-}, \cdots, V_{-}\right)=F\left(V_{+}, \cdots V_{+}\right)=0 .
$$

Proof. We only give the proof under the case $\left(\mathrm{A}_{4}^{1}\right)$ and the case $\left(\mathrm{A}_{4}^{2}\right)$ is similar. Firstly, we show

$$
\lim _{t \rightarrow-\infty} F\left(\left(g_{1} * \Phi\right)(t), \cdots,\left(g_{m} * \Phi\right)(t)\right)=F\left(V_{-}, \cdots V_{-}\right) .
$$

For fixed $j=1, \cdots, m$, let $h_{j}(x)=\int_{0}^{+\infty} g_{j}(s, x) \mathrm{d} s$, then by (1.2), we know $\int_{-\infty}^{+\infty} h_{j}(x) d x=1, j=1, \cdots, m$. Hence, $\forall \varepsilon>0\left(\varepsilon<m L, L=\max _{1 \leq j \leq m} L_{j}\right)$, there is a constant $A>0$ s.t.

$$
\left\{\begin{array}{l}
\int_{A}^{+\infty} h_{j}(x) \mathrm{d} x<(\varepsilon / m L)^{1 / \sigma} /\left(8\|\Phi\|_{0}\right) \\
\int_{-\infty}^{-A} h_{j}(x) \mathrm{d} x<(\varepsilon / m L)^{1 / \sigma} /\left(8\|\Phi\|_{0}\right),
\end{array} j=1, \cdots, m .\right.
$$

where $\sigma=\min _{1 \leq j \leq m}\left\{\sigma_{j}\right\}$. By $\left(\mathrm{A}_{4}^{1}\right)$, for the above $A$, there exists a constant $B>0$ s.t.

$$
\int_{B}^{+\infty} g_{i}(t, x) \mathrm{d} t<(\varepsilon / m L)^{1 / \sigma} /\left(16 A\|\Phi\|_{0}\right), j=1, \cdots, m .
$$

holds uniformly for $x \in[-A, A]$. Since $\lim _{t \rightarrow-\infty} \Phi(t)=V_{-}$, for the above constants $\varepsilon, \sigma, m$ and $L$, there exists an constant $T>0$ s.t.

$$
\left\|\Phi(t)-V_{-}\right\|<(\varepsilon / m L)^{1 / \sigma} / 4, t<-T .
$$

Obviously, $\left(g_{j} * \Phi\right)(t),\left(g_{j} * V_{-}\right)(t) \in[0, K], t \in \mathbb{R}$, $j=1, \cdots, m$, then by (3.2)-(3.4) and $\left(\mathrm{A}_{2}\right)$, we have

$$
\begin{aligned}
& \left\|F\left(\left(g_{1} * \Phi\right)(t), \cdots,\left(g_{m} * \Phi\right)(t)\right)-F\left(V_{-}, \cdots, V_{-}\right)\right\| \leq \sum_{j=1}^{m} L_{j}\left[\int_{0}^{+\infty} \int_{-\infty}^{+\infty} g_{i}(s, y)\left\|\Phi(t-y-c s)-V_{-}\right\| \mathrm{d} y \mathrm{~d} s\right]^{\sigma_{j}} \\
& =\sum_{j=1}^{m} L_{j}\left[\int_{-A}^{A} \int_{0}^{B} g_{j}(s, y)\left\|\Phi(t-y-c s)-V_{-}\right\| \mathrm{d} s \mathrm{~d} y+\int_{-A}^{A} \int_{B}^{+\infty} g_{j}(s, y)\left\|\Phi(t-y-c s)-V_{-}\right\| \mathrm{d} s \mathrm{~d} y\right. \\
& \left.\quad+\int_{A}^{+\infty} \int_{0}^{+\infty} g_{j}(s, y)\left\|\Phi(t-y-c s)-V_{-}\right\| \mathrm{d} s \mathrm{~d} y\right]^{\sigma_{j}} \leq \varepsilon, \\
& t<-T-A .
\end{aligned}
$$


Therefore,

$$
\begin{aligned}
& \lim _{t \rightarrow-\infty} F\left(\left(g_{1} * \Phi\right)(t), \cdots,\left(g_{m} * \Phi\right)(t)\right) \\
& =F\left(V_{-}, \cdots, V_{-}\right),
\end{aligned}
$$

similarly,

$$
\begin{aligned}
& \lim _{t \rightarrow \infty} F\left(\left(g_{1} * \Phi\right)(t), \cdots,\left(g_{m} * \Phi\right)(t)\right) \\
& =F\left(V_{+}, \cdots, V_{+}\right) .
\end{aligned}
$$

For the $i$ that $d_{i}>0$, we denote $B_{i}:=\lim _{t \rightarrow \infty} \sup \phi_{i}^{\prime}(t)$ $b_{i}:=\liminf _{t \rightarrow \infty} \phi_{i}^{\prime}(t), i=1, \cdots, n$, then $B_{i}>b_{i}$. We claim $B_{i}=$ $b_{i}$, otherwise $B_{i}>b_{i}$, then by Lemma 2.1 we know there are sequences $\left\{s_{N}\right\}_{N=1}^{\infty}$ and $\left\{t_{N}\right\}_{N=1}^{\infty}$ with

$$
\begin{aligned}
\lim _{N \rightarrow \infty} s_{N} & =\lim _{N \rightarrow \infty} t_{N}=\infty \text { s.t. } \\
& \left\{\begin{array}{l}
\lim _{N \rightarrow \infty} \phi_{i}^{\prime}\left(s_{N}\right)=\liminf _{t \rightarrow \infty} \phi_{i}^{\prime}(t) \text { and } \phi_{i}^{\prime \prime}\left(s_{N}\right)=0, \\
\lim _{N \rightarrow \infty} \phi_{i}^{\prime}\left(t_{N}\right)=\lim _{t \rightarrow \infty} \sup \phi_{i}^{\prime}(t) \text { and } \phi_{i}^{\prime \prime}\left(t_{N}\right)=0 .
\end{array}\right.
\end{aligned}
$$

Substituting $\left\{s_{N}\right\}_{N=1}^{\infty}$ and $\left\{t_{N}\right\}_{N=1}^{\infty}$ the ith equation of (2.1), we have $c B_{i}=f_{i}\left(V_{+}, \cdots, V_{+}\right), c b_{i}=f_{i}\left(V_{+}, \cdots, V_{+}\right)$, as $N \rightarrow \infty$. This contradicts with $B_{i}>b_{i}$, by the monotonicity and boundedness of $\phi_{i}(t), \lim _{t \rightarrow \infty} \phi_{i}^{\prime}(t) \geq 0$ exists (finite). From the ith equation of (2.1), we also have $\lim _{t \rightarrow \infty} \phi_{i}^{\prime \prime}(t)=\frac{c}{d_{i}} \lim _{t \rightarrow \infty} \phi_{i}^{\prime}(t)-\frac{1}{d_{i}} f_{i}\left(V_{+}, \cdots, V_{+}\right)$exists (finite). Similar to the proof of the uniformly continuity of $\Phi(t)$ in Proposition 3.1, we can obtain that $\phi_{i}^{\prime}(t)$ is uniformly continuous in $[0, \infty)$. Combining $\lim \Phi(t)=V_{+}$and Lemma 2.2, $\lim _{t \rightarrow \infty} \phi_{i}^{\prime}(t)=0$, then $\lim _{t \rightarrow \infty} \phi_{i}^{\prime \prime}(t)=0$. Therefore, by the ith equation of (2.1), we have

$$
f_{i}\left(V_{+}, \cdots, V_{+}\right)=\lim _{t \rightarrow \infty}\left[c \phi_{i}^{\prime}(t)-d_{i} \phi_{i}^{\prime \prime}(t)\right]=0 .
$$

For the $i$ that $d_{i}=0,(i=1, \cdots, n)$, by Proposition 3.1, we know

$$
\phi_{i}^{\prime}(t)=f_{i}\left(\left(g_{1} * \Phi\right)(t), \cdots,\left(g_{m} * \Phi\right)(t)\right) / c .
$$

Is uniformly continuous in $\mathbb{R}$. Considering $\lim _{t \rightarrow \infty} \Phi(t)=V_{+}$ is finite, by Lemma 2.2, we have $\lim _{t \rightarrow \infty} \phi_{i}^{\prime}(t)=0$. Hence,

$$
\begin{aligned}
& f_{i}\left(\left(g_{1} * V_{+}\right), \cdots,\left(g_{m} * V_{+}\right)(t)\right)=c \lim _{t \rightarrow \infty} \phi_{i}^{\prime}(t)=0 . \text { is given by } \\
& P_{i}(\Phi)(t)= \begin{cases}\left.\left[\int_{-\infty}^{t} \mathrm{e}^{\lambda_{i 1}(t-s)} H_{i}(\Phi)(s) \mathrm{d} s+\int_{t}^{+\infty} \mathrm{e}^{\lambda_{i 2}(t-s)} H_{i}(\Phi)(s) \mathrm{d} s\right] / d_{i}\left(\lambda_{i 2}-\lambda_{i 1}\right)\right] & \text { if } d_{i}>0 \\
\int_{-\infty}^{t} \mathrm{e}^{\lambda_{i 1}(t-s)} H_{i}(\Phi)(s) \mathrm{d} s / c & \text { if } d_{i}=0\end{cases}
\end{aligned}
$$

Then $F\left(V_{+}, \cdots, V_{+}\right)=0$. Similarly, $F\left(V_{-}, \cdots, V_{-}\right)=0$. The proof of Proposition 3.2 is completed.

Define $H=\left(H_{1}, \cdots, H_{n}\right)^{\mathrm{T}}: B C[0, K] \rightarrow B C_{\mu}\left(\mathbb{R} ; \mathbb{R}^{n}\right)$, s.t. $\forall \Phi \in B C[0, K], H(\Phi)=\left(H_{1}(\Phi), \cdots, H_{n}(\Phi)\right)^{\mathrm{T}}$ satisfying

$$
\begin{aligned}
& H(\Phi)(t) \\
& =F\left(\left(g_{1} * \Phi\right)(t), \cdots,\left(g_{m} * \Phi\right)(t)\right)+\gamma \Phi(t), \\
& t \in \mathbb{R} .
\end{aligned}
$$

Then we have the following lemma.

Lemma 3.1. Assume $\left(A_{1}\right)$ and $\left(A_{5}\right)$ hold, for all $\Phi$, $\Psi \in B C[0, K]$, the operator $H$ defined by (3.5) satisfies

1) $0 \leq H(\Phi)(t) \leq \gamma K, t \in \mathbb{R}$.

2) If $\Psi \leq \Phi, H(\Psi) \leq H(\Phi)$.

3) If $\Phi(t)$ is nondecreasing in $\mathbb{R}, H(\Phi)$ is also nondecreasing in $\mathbb{R}$.

Proof. 1) and 2) can be given directly by $\left(\mathrm{A}_{1}\right)$ and $\left(\mathrm{A}_{5}\right)$.

3) For $\theta>0$, let $\Psi(\theta)=\Phi(t+\theta)$ then

$$
\begin{gathered}
\left(g_{j} * \Phi\right)(t+\theta)=\left(g_{j} * \Psi\right)(t), \\
j=1, \cdots, m .
\end{gathered}
$$

By the monotonicity of $\Phi(t)$ we know $\hat{0} \leq \Phi \leq \Psi \leq$ $\hat{K}$, by 2$)$,

$$
\begin{aligned}
& H(\Phi)(t+\theta)=H(\Psi)(t) \geq H(\Phi)(t), \\
& t \in \mathbb{R},
\end{aligned}
$$

and this complete the proof of Lemma 3.1.

Without loss of generality, we assume $\gamma_{i}>0$ in $\left(\mathrm{A}_{5}\right)$, and denote

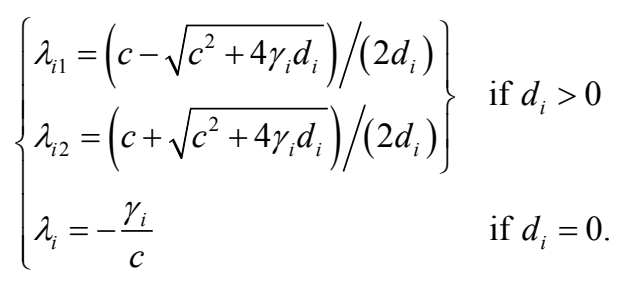

Defining the integral operator $P$ on $B C[0, K]$, $\forall \Phi \in B C[0, K], t \in \mathbb{R}$,

$$
P(\Phi)(t)=\left(P_{1}(\Phi)(t), \cdots, P_{n}(\Phi)(t)\right)^{\mathrm{T}}
$$


where $H(\Phi)=\left(H_{1}(\Phi), \cdots, H_{n}(\Phi)\right)^{\mathrm{T}}$ is defined by (3.5). Then we have the following two propositions.

Proposition 3.3. Assume (A2), (A4) and (A5) hold. The integral operator $P$ defined by (3.7) maps $B C[0, K]$ into $B C[0, K]$, and $P(\Phi) \in B C^{2}\left(\mathbb{R} ; \mathbb{R}^{n}\right)$, $\forall \Phi \in B C[0, K]$.

Proof. We only give the proof under the case $\left(\mathrm{A}_{4}^{1}\right)$, and the case $\left(\mathrm{A}_{4}^{2}\right)$ is similar.

For $\Phi \in B C[0, K]$, by Lemma 3.11 ), we have $0 \leq H(\Phi)(t) \leq \gamma K, t \in \mathbb{R}$, then, if $d_{i}>0$

$$
0 \leq P_{i}(\Phi)(t) \leq\left[\int_{-\infty}^{t} \mathrm{e}^{\lambda_{i l(t-s)}} \gamma_{i} K_{i} \mathrm{~d} s+\int_{t}^{+\infty} \mathrm{e}^{\lambda_{i 2(t-s)}} \gamma_{i} K_{i} \mathrm{~d} s\right] / d_{i}\left(\lambda_{i 2}-\lambda_{i 1}\right) \leq K_{i}
$$

If $d_{i}=0,0 \leq P_{i}(\Phi)(t) \leq \int_{-\infty}^{t} \mathrm{e}^{\lambda_{i 1}(t-s)} \mathrm{d} s \cdot \gamma_{i} K_{i} / c=K_{i}$.

Therefore, $\hat{0} \leq P(\Phi) \leq \hat{K}$. In addition, similar to the proof of Proposition 3.2 we can obtain, $\forall \varepsilon>0(\varepsilon<m L)$, there exists a constant $A>0$ s.t. for $j=1, \cdots, m$,

$$
\begin{aligned}
& \int_{A}^{+\infty} h_{j}(x) \mathrm{d} x<(\varepsilon / m L)^{1 / \sigma} / 8 \tilde{K}, \\
& \int_{-\infty}^{-A} h_{j}(x) \mathrm{d} x<(\varepsilon / m L)^{1 / \sigma} / 8 \tilde{K} .
\end{aligned}
$$

And for this $A$, there is a constant $B>0$ s.t. for $j=1, \cdots, m$,

$$
\begin{aligned}
\int_{B}^{+\infty} g_{j}(t, x) \mathrm{d} t< & (\varepsilon / m L)^{1 / \sigma} / 16 \tilde{K}, x \in[-A, A] \quad t \in \mathbb{R} \quad j=1, \cdots, m, \text { then by }\left(\mathrm{A}_{2}\right) \text { we obtain } \\
& \left\|F\left(\left(g_{1} * \Phi\right)(t+\Delta t), \cdots,\left(g_{m} * \Phi\right)(t+\Delta t)\right)-F\left(\left(g_{1} * \Phi\right)(t), \cdots,\left(g_{m} * \Phi\right)(t)\right)\right\| \\
& \leq \sum_{j=1}^{m} L_{j}\left[\int_{0}^{+\infty} \int_{-\infty}^{+\infty} g_{j}(s, y)\|\Phi(t+\Delta t-y-c s)-\Phi(t-y-c s)\| \mathrm{d} y \mathrm{~d} s\right]^{\sigma_{j}} \\
& <\sum_{j=1}^{m} L_{j}\left\{\int_{-A}^{A} \int_{0}^{B} g_{j}(s, y)(\varepsilon / m L)^{1 / \sigma} / 4 \mathrm{~d} y \mathrm{~d} s+\int_{-A}^{A} \int_{B}^{+\infty} g_{j}(s, y)(2 \tilde{K}) \mathrm{d} y \mathrm{~d} s\right. \\
& \left.+\int_{A}^{+\infty} \int_{0}^{+\infty} g_{j}(s, y)(2 \tilde{K}) \mathrm{d} y \mathrm{~d} s+\int_{-\infty}^{-A} \int_{0}^{+\infty} g_{j}(s, y)(2 \tilde{K}) \mathrm{d} y \mathrm{~d} s\right\}^{\sigma_{j}} \leq \varepsilon .
\end{aligned}
$$

Obviously, $\quad\left(g_{j}^{*} \Phi\right)(t+\Delta t), \quad\left(g_{j} * \Phi\right)(t) \in[0, K]$,

Hence, $F\left(\left(g_{1} * \Phi\right)(t), \cdots,\left(g_{m} * \Phi\right)(t)\right)$ is continuous in $\mathbb{R}$, then

$$
H(\Phi)(t)=F\left(\left(g_{1} * \Phi\right)(t), \cdots,\left(g_{m} * \Phi\right)(t)\right)+\gamma \Phi(t)
$$

is continuous in $\mathbb{R}$. In addition, by calculating directly we can obtain the following, if $d_{i}>0$,

$$
\begin{gathered}
P_{i}(\Phi)^{\prime}(t)=\left[\lambda_{i 1} \int_{-\infty}^{t} \mathrm{e}^{\lambda_{i 1}(t-s)} H_{i}(\Phi)(s) \mathrm{d} s+\lambda_{i 2} \int_{t}^{+\infty} \mathrm{e}^{\lambda_{i 2}(t-s)} H_{i}(\Phi)(s) \mathrm{d} s\right] /\left[d_{i}\left(\lambda_{i 2}-\lambda_{i 1}\right)\right] \\
P_{i}(\Phi)^{\prime \prime}(t)=\left[\lambda_{i 1}^{2} \int_{-\infty}^{t} \mathrm{e}^{\lambda_{i 1}(t-s)} H_{i}(\Phi)(s) \mathrm{d} s+\left(\lambda_{i 1}-\lambda_{i 2}\right) H_{i}(\Phi)(t)+\lambda_{i 2}^{2} \int_{t}^{+\infty} \mathrm{e}^{\lambda_{i 2}(t-s)} H_{i}(\Phi)(s) \mathrm{d} s\right] /\left[d_{i}\left(\lambda_{i 2}-\lambda_{i 1}\right)\right] .
\end{gathered}
$$

If $d_{i}=0$,

$P_{i}(\Phi)^{\prime}(t)=\left[\lambda_{i} \int_{-\infty}^{t} \mathrm{e}^{\lambda_{i}(t-s)} H_{i}(\Phi)(s) \mathrm{d} s+H_{i}(\Phi)(t)\right] / c$.

Therefore, $P(\Phi) \in B C[0, K] \cap B C^{2}\left(\mathbb{R} ; \mathbb{R}^{2}\right)$. The proof of Proposition 3.3 is completed.

Proposition 3.4. For $\Phi \in B C[0, K], \Psi \in B C^{2}\left(\mathbb{R} ; \mathbb{R}^{n}\right)$, $\Psi(t)=P(\Phi)(t)$ iff

$$
-D \Psi^{\prime \prime}(t)+c \Psi^{\prime}(t)+\gamma \Psi(t)=H(\Phi)(t),
$$

$t \in \mathbb{R}$. Especially, $\Phi \in B C[0, K]$ is a solution of wave Equation (2.1) if and only if $\Phi$ is a fixed point of $P$.
Proof. We only prove the case $d_{i}=0$, and the proof for the case $d_{i}>0$, can be given similarly. If

$$
\psi_{i}(t)=P_{i}(\Phi)(t)=\int_{-\infty}^{t} \mathrm{e}^{\lambda_{i}(t-s)} H_{i}(\Phi)(s) \mathrm{d} s / c,
$$

let $\lambda_{i}=-\gamma_{i} / c$, We obtain $c \psi_{i}^{\prime}(t)+\gamma_{i} \psi_{i}(t)=H_{i}(\Phi)(t)$. On the other hand, by the above argument,

$$
c P_{i}(\Phi)^{\prime}(t)+\gamma_{i} P_{i}(\Phi)(t)=H_{i}(\Phi)(t) .
$$

So we have $c\left[\psi_{i}-P_{i}(\Phi)\right]^{\prime}(t)+\gamma_{i}\left[\psi_{i}-P_{i}(\Phi)\right](t)=0$.

Then $\psi_{i}(t)-P_{i}(\Phi)(t)=\alpha \mathrm{e}^{\lambda_{i t}} \quad$ where $\alpha$ is a constant. 
By Proposition 3.3, we know $\forall \Phi \in B C[0, K]$, $P(\Phi) \in B C[0, K]$. Since $\Psi \in B C^{2}\left(\mathbb{R} ; \mathbb{R}^{n}\right)$, $\psi_{i}(t)-P_{i}(\Phi)(t)$ is bounded in $\mathbb{R}$, hence $\alpha=0$, then $\psi_{i}(t)=P_{i}(\Phi)(t)$. And this completes the proof of Proposition 3.4.

By Lemma 3.1, we can easily obtain the following lemma on the monotonicity of the integral operator $P$.

Lemma 3.2. Assume $\left(A_{1}\right)$ and $\left(A_{5}\right)$ hold, then

1) If $\Psi, \Phi \in B C[0, K]$, and $\Psi \leq \Phi, \quad P(\Psi) \leq P(\Phi)$.

2) If $\Phi \in B C[0, K]$ is nondecreasing in $\mathbb{R}, P(\Phi)$ is also nondecreasing in $\mathbb{R}$.

On the continuity of the integral operator $P$, we have the following.

Proposition 3.5. Assume $\left(\mathrm{A}_{2}\right)-\left(\mathrm{A}_{4}\right)$ hold. Then $P: B C[0, K] \rightarrow B C[0, K]$ is continuous with respect to the norm $\|\cdot\|_{u}$ in $B C_{u}\left(\mathbb{R} ; \mathbb{R}^{2}\right)$, where

$0<\mu<\min \left\{-\lambda_{i 1}, \lambda_{i 2},-\lambda_{i}\right\}$, and $\lambda_{i 1}, \lambda_{i 2}, \lambda_{i}$ are given by (3.6).

Proof. We first claim $H$ defined by (3.5) is continuous in $B C[0, K]$ with respect to the norm $\|\cdot\|_{u}$.

For $\Psi, \Phi \in B C[0, K]$, we know obviously $\left(g_{j} * \Phi\right)(t)$, $\left(g_{j} * \Psi\right)(t) \in B C[0, K], t \in \mathbb{R}, j=1, \cdots, m$. By $\left(\mathrm{A}_{2}\right)$ and $\left(\mathrm{A}_{3}\right)$, we obtain

$$
\begin{aligned}
\| & H(\Phi)-H(\Psi) \|_{\mu} \\
\leq & \sup _{t \in \mathbb{R}}\left\{\sum_{j=1}^{m} L_{j}\left[\|\left(g_{j} * \Phi\right)(t)-\left.\left(g_{j} * \Psi\right)(t)\right|^{\sigma_{j}}\right] \mathrm{e}^{-u|t|}\right\} \\
& +\tilde{\gamma} \max _{1 \leq i \leq n} \sup _{t \in \mathbb{R}}\left\{\left|\phi_{i}(t)-\psi_{i}(t)\right| \mathrm{e}^{-\mu|t|}\right\} \\
\leq & \sup _{t \in \mathbb{R}}\left\{\sum_{j=1}^{m} L_{j}\left[\beta \mathrm{e}^{\mu \mid t}\|\Phi-\Psi\|_{\mu}\right]^{\sigma_{j}} \mathrm{e}^{-\mu t \mid t}\right\}+\tilde{\gamma}\|\Phi-\Psi\|_{\mu} \\
\leq & {\left[\sum_{j=1}^{m} L_{j} \beta^{\sigma_{j}}+\tilde{\gamma}\right]\|\Phi-\Psi\|_{\mu}^{\sigma},\left(\text { Suppose }\|\Phi-\Psi\|_{\mu} \leq 1\right) }
\end{aligned}
$$

where $\sigma=\min _{1 \leq j \leq m}\left\{\sigma_{j}\right\} \leq 1, \quad \tilde{\gamma}=\max _{1 \leq j \leq n}\left\{\gamma_{i}\right\}$. Thus $\forall \varepsilon>0$, choose

$$
\delta=\min \left\{\left[\varepsilon\left(\sum_{j=1}^{m} L_{j} M^{\sigma_{j}}+\tilde{\gamma}\right)^{-1}\right]^{1 / \sigma}, 1\right\}
$$

then we have $\|H(\Phi)-H(\Psi)\|_{\mu}<\varepsilon$ for $\|\Phi-\Psi\|_{\mu}<\delta$, i.e., $H$ is continuous in $B C[0, K]$ with respect to the norm $\|\cdot\|_{u}$.

Now, we show $P: B C[0, K] \rightarrow B C[0, K]$ is continuous with respect to the norm $\|\cdot\|_{u}$. For $\Phi, \Psi \in B C[0, K]$, similar to the method in $\mathrm{Ma}$ [4], we obtain there is an constant $G>0$ s.t.

$$
\|P(\Phi)-P(\Psi)\|_{\mu} \leq G\|H(\Phi)-H(\Psi)\|_{\mu}
$$

By the continuity of $H$, we know $P$ is continuous with respect tothe norm $\|\cdot\|_{u}$. The proof is completed.

In the following, we state and prove the main theorem of this paper.

Theorem 3.1. Assume $\left(A_{1}\right)-\left(A_{5}\right)$ hold. Suppose wave equations (2.1) has a pair of upper and lower solution $\bar{\rho}, \underline{\rho} \in B C[0, K]$ satisfying

1) $\sup _{s \leq t} \underline{\rho}(s) \leq \bar{\rho}(t)\left(\right.$ or $\left.\underline{\rho}(t) \leq \inf _{s \geq t} \bar{\rho}(s)\right), t \in \mathbb{R}$.

2) $F(V, \cdots, V) \neq 0, V \in\left(0, \inf _{t \in \mathbb{R}} \bar{\rho}(t)\right] \cup\left[\sup _{t \in \mathbb{R}} \underline{\rho}(t), K\right)$.

Then (2.1) and (2.3) have a monotone solution, i.e., (1.1) has a traveling wavefront solution.

To prove Theorem 3.1, we define the following profile set

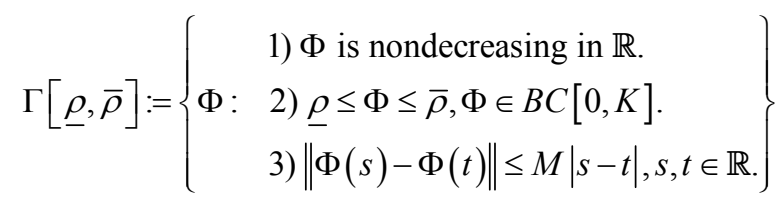

where $M=\max \left\{\gamma_{i} K_{i} / c\right\}$, we first prove two lemmas.

Lemma 3.3. . If $^{1}$ If the conditions of Theorem 3.1 hold, then for $\Phi \in C\left(\mathbb{R} ; \mathbb{R}^{n}\right)$ with $\underline{\rho} \leq \Phi \leq \bar{\rho}$, we have $\rho \leq P(\Phi) \leq \bar{\rho}$.

Proof. For $t \in \mathbb{R}$, we denote

$$
\begin{aligned}
& W(t)=\left(w_{1}(t), \cdots, w_{n}(t)\right)^{\mathrm{T}} \\
& W(t)=\left(\left(P_{1}(\Phi)(t)-\underline{\rho}_{1}(t)\right), \cdots,\left(P_{n}(\Phi)(t)-\underline{\rho}_{n}(t)\right)\right)^{\mathrm{T}}
\end{aligned}
$$

then we have $W \in B C\left(\mathbb{R} ; \mathbb{R}^{n}\right)$. In order to obtain $P(\Phi) \geq \rho$, it suffices to prove $W(t) \geq 0$.

By Proposition 3.4, we know if $d_{i}=0$,

$$
c P_{i}(\Phi)^{\prime}(t)+\gamma_{i} P_{i}(\Phi)(t)=H_{i}(\Phi)(t), t \in \mathbb{R} .
$$

From the definition of lower solution, we know

$$
c \underline{\rho}_{i}^{\prime}(t)+\gamma_{i} \underline{\rho}_{i}(t) \leq H_{i}(\underline{\rho})(t) \text {, a.e. } t \in \mathbb{R} .
$$

Considering $\Phi \geq \rho$ and by Lemma 3.12 ), we obtain

$$
\begin{gathered}
c\left[P_{i}(\Phi)(t)-\underline{\rho}_{i}(t)\right]^{\prime}+\gamma_{i}\left[P_{i}(\Phi)(t)-\underline{\rho}_{i}(t)\right] \geq 0, \\
\text { a.e. } t \in \mathbb{R} .
\end{gathered}
$$

Let

$$
r_{i}(t)=c w_{i}^{\prime}(t)+\gamma_{i} w_{i}(t), t \in \mathbb{R} .
$$

Then $r_{i}(t) \geq 0$. By the continuity of $w_{i}(t)$ and formula of variant of constants, we have

$$
w_{i}(t)=\alpha \mathrm{e}^{\lambda_{i} t}+\int_{-\infty}^{t} \mathrm{e}^{\lambda_{i}(t-s)} r_{i}(s) \mathrm{d} s,
$$

where $\alpha$ is a constant. By Lemma 3.1, we know $P_{i}(\Phi)$, $P_{i}(\Phi)^{\prime}$ are both bounded, It follows by (3.8) that $w_{i}(t)$, $w_{i}^{\prime}(t)$ are essentially bounded in $\mathbb{R}$, then by (3.9), 
$r_{i}(t)$ is also essentially bounded in $\mathbb{R}$, so $\alpha=0$ in (3.10), and $P_{i}(\Phi) \geq \rho_{i}, \quad\left(\right.$ for $\left.d_{i}=0\right)$. In a similar way, we can obtain $P_{i}(\Phi) \Sigma \rho_{i}$, (for $\left.d_{i}>0\right)$ therefore $P(\Phi) \geq$ $\rho$. Similarly, we can prove $P(\Phi) \leq \bar{\rho}$. The proof is completed.

Lemma 3.4. If the conditions of Theorem 3.1 hold, then $P$ is equi-continuous.

Proof. For $\Phi \in B C[0, K]$, if $d_{i}>0$, by Lemma 3.1 1) and Proposition 3.3, we have, for $t \in \mathbb{R}$,

$$
P_{i}(\Phi)^{\prime}(t) \geq \frac{\lambda_{i 1}}{d_{i}\left(\lambda_{i 2}-\lambda_{i 1}\right)} \int_{-\infty}^{t} \mathrm{e}^{\lambda_{i 1}(t-s)}\left(\gamma_{i} K_{i}\right) \mathrm{d} s>-\frac{\gamma_{i} K_{i}}{c},
$$

and,

$$
P_{i}(\Phi)^{\prime}(t) \leq \frac{\lambda_{i 2}}{d_{i}\left(\lambda_{i 2}-\lambda_{i 1}\right)} \int_{t}^{+\infty} \mathrm{e}^{\lambda_{i 2}(t-s)}\left(\gamma_{i} K_{i}\right) \mathrm{d} s<\frac{\gamma_{i} K_{i}}{c},
$$

then, $\left|P_{i}(\Phi)^{\prime}(t)\right|<\gamma_{i} K_{i} / c$, this is also holds for the case $d_{i}=0$, the proof is similar and we omit here.

$\forall \varepsilon>0$, we choose $\delta=\varepsilon / M$, here $M=\max _{1 \leq i \leq n}\left\{\gamma_{i} K_{i} / c\right\}$, then for $P(\Phi) \in P(B C[0, K])$, by Lagrange theorem, if $t_{1}, t_{2} \in \mathbb{R},\left|t_{1}-t_{2}\right|<\delta$,

$$
\left|P_{i}(\Phi)\left(t_{1}\right)-P_{i}(\Phi)\left(t_{2}\right)\right|=\left|P_{i}(\Phi)^{\prime}\left(\eta_{i}\right)\right|\left|t_{1}-t_{2}\right| \leq M\left|t_{1}-t_{2}\right|,
$$

$\eta_{i} \in\left(t_{1}, t_{2}\right)$,

then $\left\|P(\Phi)\left(t_{1}\right)-P(\Phi)\left(t_{2}\right)\right\|<\varepsilon$, i.e., $P(B C[0, K])$ is equi-continuous. This completes the proof of Lemma 3.4.

Proof of Theorem 3.1. We divide the proof of Theorem 3.1 into five steps.

Step $1, \Gamma[\underline{\rho}, \bar{\rho}]$ is a nonempty and convex set.

1) Denote $\tilde{\Phi}(t)=\sup _{s \leq t} \underline{\rho}(s)\left(\right.$ or $\left.\tilde{\Phi}(t)=\inf _{s \geq t} \bar{\rho}(s)\right), t \in \mathbb{R}$.

Obviously, $\tilde{\Phi}$ is continuous and nondecreasing in $\mathbb{R}$, then by Lemma 3.21$), P(\tilde{\Phi})$ is nondecreasing in $\mathbb{R}$.

2) By Theorem 3.1(a), we know $\hat{0} \leq \rho \leq \tilde{\Phi} \leq \bar{\rho} \leq \hat{K}$, then by lemma 3.21 ) and Lemma 3.3 we have

$$
\underline{\rho} \leq P(\underline{\rho}) \leq P(\tilde{\Phi}) \leq P(\bar{\rho}) \leq \bar{\rho} .
$$

3) By the above 1) and 2) we also know $\tilde{\Phi} \in B C[0, K]$, similar to the proof of Lemma 3.4 ,we obtain

$$
\left\|P(\tilde{\Phi})\left(t_{1}\right)-P(\tilde{\Phi})\left(t_{2}\right)\right\| \leq M\left|t_{1}-t_{2}\right|, \eta_{i} \in\left(t_{1}, t_{2}\right) .
$$

Therefore, $P(\tilde{\Phi}) \in \Gamma[\underline{\rho}, \bar{\rho}]$, then $\Gamma[\underline{\rho}, \bar{\rho}]$ is nonempty. It is obvious that $\Gamma[\underline{\rho}, \bar{\rho}]$ is convex.

Step 2, $\Gamma[\underline{\rho}, \bar{\rho}]$ is a closed set in $B C_{\mu}\left(\mathbb{R} ; \mathbb{R}^{n}\right)$, i.e., if sequences

$$
\left\{\Phi^{k}\right\}_{k=1}^{\infty}=\left\{\left(\phi_{1}^{k}, \cdots, \phi_{n}^{k}\right)^{\mathrm{T}}\right\}_{k=1}^{\infty} \subset \Gamma[\underline{\rho}, \bar{\rho}]
$$

converge to $\Phi$ with respect to the norm $\|\cdot\|_{\mu}$, then $\Phi \in \Gamma[\underline{\rho}, \bar{\rho}]$. Since

$$
\left\|\Phi^{k}-\Phi\right\|_{\mu}=\max _{1 \leq i \leq n} \sup _{t \in \mathbb{R}}\left\{\left|\phi_{i}^{k}(t)-\phi_{i}\right| \mathrm{e}^{-\mu|t|}\right\} \rightarrow 0,
$$

for the fixed $t$

$$
\left\|\Phi^{k}(t)-\Phi(t)\right\| \leq\left\|\Phi^{k}-\Phi\right\|_{\mu} \mathrm{e}^{\mu|t|} \rightarrow 0, \text { as } k \rightarrow \infty .
$$

$\forall \varepsilon>0$, we choose $\delta=\varepsilon / 3 M$, then by

$\left\{\Phi^{k}\right\}_{k=1}^{\infty} \subset \Gamma[\underline{\rho}, \bar{\rho}]$ we know

$\left|\Phi^{k}(t)-\Phi^{k}(t+\Delta t) \|<\varepsilon / 3,\right| \Delta t \mid<\delta, t \in \mathbb{R}, k=1,2, \cdots$. By (3.11), there is a $N$ s.t. for the above $t, \Delta t$

$$
\left\|\Phi^{N}(t)-\Phi(t)\right\|<\varepsilon / 3,
$$

and

$$
\left\|\Phi^{N}(t+\Delta t)-\Phi(t+\Delta t)\right\|<\varepsilon / 3 .
$$

Therefore, for fixed $t \in \mathbb{R}$, if $|\Delta t|<\delta$,

$$
\begin{aligned}
& \|\Phi(t)-\Phi(t+\Delta t)\| \\
& \leq\left\|\Phi^{N}(t)-\Phi(t)\right\|+\left\|\Phi^{N}(t)-\Phi^{N}(t+\Delta t)\right\| \\
& \quad+\left\|\Phi^{N}(t+\Delta t)-\Phi(t+\Delta t)\right\|<\varepsilon
\end{aligned}
$$

i.e., $\Phi(t)$ is continuous in $\mathbb{R}$. In addition, we have

1) $\forall t_{1}, t_{2} \in \mathbb{R}, t_{1} \leq t_{2}$, since $\Phi^{k}\left(t_{1}\right) \leq \Phi^{k}\left(t_{2}\right)$, $k=1,2, \cdots$, by $(3.11)$,

$$
\Phi\left(t_{1}\right)-\Phi\left(t_{2}\right) \leq \Phi^{k}\left(t_{1}\right)-\Phi\left(t_{1}\right)+\Phi^{k}\left(t_{2}\right)-\Phi\left(t_{2}\right) \leq 0,
$$

i.e., $\Phi(t)$ is continuous in $\mathbb{R}$.

2) $\forall t \in \mathbb{R}$, since $\Phi^{k}(t) \leq \bar{\rho}(t), k=1,2, \cdots$, by (3.11), $\Phi(t)-\bar{\rho}(t) \leq \Phi(t)-\Phi^{k}(t) \leq 0$. Similarly $\Phi \geq \underline{\rho}$, so $\Phi \in B C[0, K]$.

3) Since $\left\|\Phi^{k}(s)-\Phi^{k}(t)\right\| \leq M|s-t|, \quad s, t \in \mathbb{R}$, $k=1,2, \cdots$,

$$
\begin{aligned}
& \|\Phi(s)-\Phi(t)\| \\
& \leq\left\|\Phi^{k}(s)-\Phi(s)\right\|+M|s-t|+\left\|\Phi^{k}(t)-\Phi(t)\right\|,
\end{aligned}
$$

combining with (3.11), we obtain $\|\Phi(s)-\Phi(t)\| \leq M|s-t|$. Therefore, $\Phi \in \Gamma[\underline{\rho}, \bar{\rho}]$.

Step 3, $P(\Gamma[\underline{\rho}, \bar{\rho}]) \subset \Gamma[\underline{\rho}, \bar{\rho}]$. This can be easily proved followed by Proposition 3.3 and Lemma 3.2 - 3.4.

Step 4, $P(\Gamma[\underline{\rho}, \bar{\rho}])$ is sequentially compact.

For $\Phi \in \Gamma[\underline{\rho}, \bar{\rho}]$. by Proposition 3.3 we know $P(\Phi) \in B C[0, K]$, then $\|P(\Phi)\|_{0} \leq \tilde{K}$, i.e., $P(\Gamma[\underline{\rho}, \bar{\rho}])$ is uniformly bounded. It follows by Lemma 3.4 that $P(\Gamma[\underline{\rho}, \bar{\rho}])$ is equi-continuous. We define two operators

$$
P_{N}=\left(P_{N 1}, \cdots, P_{N n}\right)^{\mathrm{T}}: B C[0, K] \rightarrow B C[0, K]
$$

and

$$
Q_{N}=\left(Q_{1}, \cdots, Q_{N n}\right)^{\mathrm{T}}: B C[0, K] \rightarrow C\left([-N, N] ; \mathbb{R}^{n}\right)
$$

satisfying 


$$
P_{N}(\Phi)(t)=\left\{\begin{array}{l}
P(\Phi)(N), t \in(N,+\infty) \\
P(\Phi)(t), t \in[-N, N] \\
P(\Phi)(-N), t \in(-\infty,-N)
\end{array}\left|Q_{N}(\Phi)(t)=\Phi(t)\right|_{[-N, N]},\right.
$$

where $N \in \mathbb{N}$. For $P(\Phi) \in P(\Gamma[\underline{\rho}, \bar{\rho}]) \subset B C^{\prime}[0, K]$, we have $\left\|P_{N}(\Phi)-P(\Phi)\right\|_{\mu} \leq 2 \tilde{K} e-\mu N$, therefore $\forall \varepsilon>$ 0 , there is an constant $\tilde{N} \in \mathbb{N}$, s.t. $\left\|P_{\tilde{N}}(\Phi)-P(\Phi)\right\|_{\mu}<\frac{\varepsilon}{3}$, $\forall \Phi \in \Gamma[\rho, \bar{\rho}]$. To the $\tilde{N}$, by the above argument, $Q_{\tilde{N}}(P(\Gamma[\underline{\rho}, \bar{\rho}]))$ is uniformly bounded and equi-continuous in $[-N, N]$, then by Arzela-Ascoli theorem, $Q_{\tilde{N}}(P(\Gamma[\underline{\rho}, \bar{\rho}]))$ is sequentially compact in $\left(C\left([-N, N], \mathbb{R}^{n}\right),\|\cdot\|\right)$. For the $\varepsilon, Q_{\tilde{N}}(P(\Gamma[\underline{\rho}, \bar{\rho}]))$ has a finite $\varepsilon / 3-$ net, we denote this $\varepsilon / 3$ - net by $\left\{Q_{\tilde{N}}\left(P\left(\Phi_{j}\right)\right)\right\}_{j=1}^{J}$, where $\Phi_{j} \in \Gamma[\underline{\rho}, \bar{\rho}], j=1, \cdots, J$, i.e.,

$$
\min _{1 \leq j \leq J}\left\|Q_{\tilde{N}}(P(\Phi))-Q_{\tilde{N}}\left(P\left(\Phi_{j}\right)\right)\right\| \leq \varepsilon / 3, \forall \Phi \in \Gamma[\underline{\rho}, \bar{\rho}] .
$$

Thus

$$
\begin{aligned}
& \min _{1 \leq j \leq J}\left\|P(\Phi)-P(\Phi)_{j}\right\|_{\mu} \\
& \leq \min _{1 \leq j \leq J}\left\|P_{\tilde{N}}(\Phi)-P_{\tilde{N}}\left(\Phi_{j}\right)\right\|_{\mu}+\left\|P_{\tilde{N}}(\Phi)-P(\Phi)\right\|_{\mu} \\
& \quad+\max _{1 \leq j \leq J}\left\|P_{\tilde{N}}\left(\Phi_{j}\right)-P\left(\Phi_{j}\right)\right\|_{\mu} \\
& \leq \min _{1 \leq j \leq J}\left\{\max _{1 \leq i \leq n} \sup \left|P_{\tilde{N}_{i}}(\Phi)(t)-P_{\tilde{N}_{i}}\left(\Phi_{j}\right)(t)\right|\right\} \\
& \quad+\left\|P_{\tilde{N}}(\Phi)-P(\Phi)\right\|_{\mu}+\max _{1 \leq j \leq J}\left\|P_{\tilde{N}}\left(\Phi_{j}\right)-P\left(\Phi_{j}\right)\right\|_{\mu} \leq \varepsilon, \\
& \forall \Phi \in \Gamma[\underline{\rho}, \bar{\rho}] .
\end{aligned}
$$

Then $\left\{P\left(\Phi_{j}\right)\right\}_{j=1}^{J}$ is the finite $\varepsilon$-net of $P(\Gamma[\underline{\rho}, \bar{\rho}])$ and so $P(\Gamma[\underline{\rho}, \bar{\rho}])$ is sequentially compact.

Step 5, (2.1) and (2.3) have a monotone solution.

By Proposition 3.5, we know $P: \Gamma[\underline{\rho}, \bar{\rho}] \rightarrow \Gamma[\underline{\rho}, \bar{\rho}]$ is continuous with respect to the norm solution.

By Proposition 3.5, we know $P: \Gamma[\underline{\rho}, \bar{\rho}] \rightarrow \Gamma[\underline{\rho}, \bar{\rho}]$ is continuous with respect to the norm $\|\cdot\|_{\mu}$, combining with step $1-4, P$ satisfies all conditions of Schauder fixed point theorem in $\Gamma[\rho, \bar{\rho}]$, therefore $P$ has fixed point $\Theta$ in $\Gamma[\rho, \bar{\rho}]$, by Proposition $3.4, \Theta$ is the monotone solution of (2.1) and (2.3). Since

$\Theta \in \Gamma[\underline{\rho}, \bar{\rho}] \subset B C[0, K]$ is monotone and bounded in $\mathbb{R}, \quad V_{-}:=\lim _{t \rightarrow-\infty} \Theta(t)$ and $V_{+}:=\lim _{t \rightarrow-\infty} \Theta(t)$ exists, by Propo- sition 3.2, $F\left(V_{-}, \cdots, V_{-}\right)=F\left(V_{+}, \cdots, V_{+}\right)=0$. It follows by $\Theta \in \Gamma[\underline{\rho}, \bar{\rho}]$ that $\underline{\rho} \leq \Theta \leq \bar{\rho}, 0 \leq V_{-} \leq \inf _{t \in \mathbb{R}} \bar{\rho}(t)$, and $\sup _{t \in \mathbb{R}} \underline{\rho}(t) \leq V_{+} \leq K$. Then by $\left(\mathrm{A}_{1}\right)$ and condition (b) of Theorem 3.1, $V_{-}=0, V_{+}=K$, i.e., $\Theta$ satisfies the asymptotic boundary condition (2.3). Therefore (1.1) has a wave front $U(t, x)=\Theta(x+c t)$. And this completes the proof of Theorem 3.1.

\section{Conclusion}

In this paper, we study reaction-diffusion systems with spatio-temporal delays, and obtain the existence of traveling wave fronts by using Schauder's Fixed Point Theorem. In our results, we reduce the existence of traveling wave fronts to the existence of an admissible pair of upper solution and lower solution, which are much easier to construct in practice.

\section{Acknowledgements}

This research is supported by Jiangsu Province Natural Science Research Projects (No.12KJB110017), NUPT's Scientific Fund (No. NY208028, NY211140) and NUAA's Scientific Fund (No. NS2011001, NN2012048).

\section{REFERENCES}

[1] S. Ai, "Traveling Wave Fronts for Generalized Fisher Equations with Spatio-Temporal Delays," Journal of Differential Equations, Vol. 232, No. 1, 2007, pp. 104-133.

[2] V. Capasso and L. Maddalena, "Convergence to Equilibrium States for a Reaction-Diffusion System Modelling the Spatial Spread of a Class of Bacterial and Viral Disease," Journal of Mathematical Biology, Vol. 13, No. 2, 1981, pp. 173-184. doi:10.1007/BF00275212

[3] K. Gopalsamy, "Stability and Oscillations in Delay Difference Differential Equations of Population Dynamics," Kluwer Academic, Dordrecht, 1992. doi:10.1007/978-94-015-7920-9

[4] S. Ma, "Traveling Wavefronts for Delayed Reaction-Diffusion Systems via a Fixed Point Theorem," Journal of Differential Equations, Vol. 171, No. 2, 2001, pp. 294 314.

[5] P. Popivanov, A. Slavova and P. Zecca, "Compact Traveling Waves and Peakon Type Solutions of Several Equations of Mathematical Physics and Their Cellular Neural Network Realization," Nonlinear Analysis: Real World Applications, Vol. 10, No. 3, 2009, pp. 1453-1465.

[6] K. Schaaf, "Asymptotic Behavior and Traveling Wave 
Solutions for Parabolic Functional Differential Equations," Transactions of the American Mathematical Society, Vol. 302, 1987, pp. 587-615.

[7] Z. Wang, W. Li and S. Ruan, "Traveling Wave Fronts in Reaction-Diffusion Systems with Spatio-Temporal Delays," Journal of Differential Equations, Vol. 222, No. 1, 2006, pp. 185-232. doi:10.1016/j.jde.2005.08.010

[8] J. Wu and X. Zou, "Traveling Wave Fronts of ReactionDiffusion Systems with Delays," Journal of Dynamics and Differential Equations, Vol. 13, No. 3, 2001, pp. 651687.

[9] X. Zou and J. Wu, "Existence of Traveling Wavefronts in Delayed Reaction-Diffusion System via Monotone Iteration Method," Proceedings of the American Mathematical Society, Vol. 125, 1997, pp. 2589-2598. doi:10.1090/S0002-9939-97-04080-X

[10] D. Xu and X. Zhao, "Bistable Waves in an Epidemic Model," Journal of Dynamics and Differential Equations, Vol. 16, No. 3, 2004, pp. 679-707. doi:10.1007/s10884-004-6113-Z

[11] X. Zhao and W. Wang, "Fisher Waves in an Epidemic Model," Discrete and Continuous Dynamical SystemsSeries B, Vol. 4, No. 4, 2004, pp. 1117-1128. doi:10.3934/dcdsb.2004.4.1117

[12] X. Zhao and D. Xiao, "The Asymptotic Speed of Spread and Traveling Waves for a Vector Disease Model," Journal of Dynamics and Differential Equations, Vol. 18, No. 4, 2006, pp. 1001-1019. doi:10.1007/s10884-006-9044-Z
[13] W. F. Yan and R. Liu, "Existence and Critical Speed of Traveling Wave Fronts in a Modified Vector Disease Model with Distributed Delay," Journal of Dynamical and Control Systems, Vol. 18, No. 3, 2012, pp. 355-378. doi:10.1007/s10883-012-9148-1

[14] G. Lin, W. T. Li and S. G. Ruan, "Asymptotic Stability of Monostable Wavefronts in Discrete-Time Integral Recursions," Science China Mathematics, Vol. 53, No. 5, 2010, pp. 1185-1194.

[15] Z.-Q. Xu and P.-X. Weng, "Traveling Waves in Nonlocal Diffusion Systems with Delays and Partial Quasi-Monotonicity," Applied Mathematics-A Journal of Chinese Universities, Vol. 26, No. 4, 2011, pp. 464-482. doi:10.1007/s11766-011-2727-1

[16] S.-L. Wu, H.-Q. Zhao and S.-Y. Liu, "Asymptotic Stability of Traveling Waves for Delayed Reaction-Diffusion Equations with Crossing-Monostability," Zeitschrift für Angewandte Mathematik und Physik (ZAMP), Vol. 62, No. 3, 2011, pp. 377-397. doi:10.1007/s00033-010-0112-1

[17] H. Y. Wang, "Spreading Speeds and Traveling Waves for Non-cooperative Reaction-Diffusion Systems," Journal of Nonlinear Science, Vol. 21, No. 5, 2011, pp. 747-783. doi:10.1007/s00332-011-9099-9

[18] X. J. Li, "Existence of Traveling Wavefronts of Nonlocal Delayed Lattice Differential Equations," Journal of Dynamical and Control Systems, Vol. 17, No. 3, 2011, pp. 427-449. doi:10.1007/s10883-011-9124-1 